\title{
Fractional Flow Reserve of Vessel
}

National Cancer Institute

\section{Source}

National Cancer Institute. Fractional Flow Reserve of Vessel. NCI Thesaurus. Code C99533.

A lesion-specific measure of stenosis severity within a blood vessel. 